\title{
ON APPROXIMATION BY FUNCTIONS OF LESSER VALENCE
}

\author{
O. J. FARRELL AND M. F. BOLSTER
}

1. Introduction. Let $G$ be a simply connected region in the plane of the complex variable $z$. The set of functions analytic in $G$ and either identically constant or $h$-valent at most in $G$ form a closed set $H$. That is to say, any limit in $G$ (uniform on every closed set in $G$ ) of functions of the family also belongs to $H$ [2, p. 8].

Let $w=f(z)$ be analytic and uniformly limited and $k$-valent in $G$. For every function $h(z)$ uniformly limited and of class $H$ we set

$$
\lambda=1 . u . b .[|h(z)-f(z)|, z \text { in } G] .
$$

Let $L(h)$ denote the greatest lower bound of all numbers $\lambda$. It follows by the theory of normal families $[4, \S \S 10,18]$ that there exists at least one function in $H$ for which $\lambda=L(h)$. Such an extremal function will be denoted by $h^{*}(z)$.

It will be understood throughout this paper that $h<k$. Then $L(h)$ must be positive. For if $L(h)$ were zero, there would exist a sequence $h_{1}(z), h_{2}(z), \cdots$ of functions in $H$ whose corresponding $\lambda$-sequence $\lambda_{1}, \lambda_{2}, \cdots$ would converge to zero with the result that the sequence $h_{1}(z), h_{2}(z), \ldots$ would converge in $G$ (uniformly on every closed set in $G)$ to $f(z)$. But this is impossible since the limit of such a sequence is at most $h$-valent in $G$.

Walsh has proved $\left[1\right.$, p. 345] that if $f(z)=z^{k}$, where $k$ is a positive integer and $G$ is the region $|z|<1$, then for every $h<k$ the value of $L(h)$ is unity. He has also found extremal functions $h^{*}(z)$ for such an $f(z)$ and such a region $G$. Our object in the present paper is to add a few results to those of Walsh. Our Theorem 1 is an appraisal on $L(h)$. In Theorem 2 the precise value of $L(h)$ is found for a restricted situation. Theorems 3 and 4 are initial results on the relation between the extremal functions and the character of the Riemann configuration $R$ onto which $G$ is mapped by $w=f(z)$.

2. Radius of $j$-coverage. Let $w=f(z)$ be analytic and uniformly limited and $k$-valent in a simply connected region $G$. Let $R$ denote the Riemann configuration over the $w$-plane onto which $G$ is mapped by $w=f(z)$. With every finite point $w_{0}$ of the $w$-plane we associate a non-negative real number $M_{j}\left(w_{0}\right)$ called the radius of $j$-coverage at $w_{0}$ (more exactly, the radius of precise $j$-coverage) as follows: 1961.

Received by the editors November 28, 1960 and, in revised form, January 18, 
(a) if there exists a positive $r$ such that for every point $w$ in the region $\left|w-w_{0}\right|<r$ there are precisely $j$ points of $R$ having affix $w$ (a branch point of $R$ of order $p-1$ counting as $p$ points of $R$ ), then we define $M_{j}\left(w_{0}\right)$ to be the largest $r$ for which there is such precise $j$-coverage of the region $\left|w-w_{0}\right|<r$.

(b) if there exists no positive $r$ such that for every $w$ in the region $\left|w-w_{0}\right|<r$ there are precisely $j$ points of $R$ having affix $w$, we define $M_{j}\left(w_{0}\right)$ to be zero.

Verification of the statement that there is a largest $r$ in (a) can be made in the manner indicated by Seidel and Walsh in their definition of radius of $p$-valence $[3, p .162]$. It is to be noted, however, that while their radius of $p$-valence is associated with each point of the Riemann configuration $R$, our radius of $j$-coverage is associated with each finite point of the w-plane.

Let $M_{j}$ denote the least upper bound for all $M_{j}(w)$. For each $j$ there is at least one $w$ at which $M_{j}(w)=M_{j}$. We denote by $M_{j}^{*}$ the greatest number to be found among those $M_{i}$ for which $i \geqq j$.

3. Circular hull and circular kernels. Let $A$ denote a limited plane point set. The reader will readily verify the existence of a unique minimal closed circular region $Q$ containing $A . Q$ is the circular hull of $A[5$, p. 96].

If $E$ is a limited plane point set having interior points, a maximal open circular region $K$ contained in $E$ is a circular kernel of $E$ [5, p. 96]. A set $E$ may have more than one circular kernel. The region bounded by two concentric circles has infinitely many circular kernels.

4. Appraisal on $L(h)$. Let $w=f(z)$ be analytic and uniformly limited and $k$-valent in a simply connected region $G$. Let $R$ denote the Riemann configuration over the w-plane onto which $G$ is mapped by the function $w=f(z)$. Let $S$ denote the projection of $R$ on the $w$-plane. That is to say, $S$ shall denote the set of those points in the w-plane over each of which lies at least one point of $R$. Denote by $S_{j}$ the set of points $w$ having positive radius of $j$-coverage. Except for the trivial case where $f(z)$ is identically constant in $G$, the projection of $R$ is a region (not necessarily simply connected). The radius of circular kernels of $S_{j}$ is $M_{j}$. Finally, let $r^{*}$ denote the radius of the circular hull of $S$. Then we have

THEOREM 1. Let $w=f(z)$ be analytic and uniformly limited and $k$ valent in a simply connected region $G$. Then the measure $L(h)$ of best approximation to $f(z)$ in $G$ by functions of the set $H$ is bounded above 
by the radius $r^{*}$ of the circular hull of $S$ and is bounded below by the largest value to be found among the radii of circular kernels of the projections $S_{h+1}, S_{h+2}, \cdots, S_{k}$. That is to say, $M_{h+1}^{*} \leqq L(h) \leqq r^{*}$.

PRoof. Let $w^{*}$ denote the center of the circular hull of $S$. For the function $g(z) \equiv w^{*}$ of class $H$ we have $\lambda=r^{*}$. Consequently $L(h)$ does not exceed $r^{*}$.

It remains to prove that $L(h)$ is not less than $M_{h+1}^{*}$. Suppose $L(h)=M^{\prime}<M_{h+1}^{*}$. In accordance with the definition of $M_{h+1}^{*}$ there exists a point $w_{0}$ together with a positive integer $j_{0}$ not less than $h+1$ such that $M_{j_{0}}\left(w_{0}\right)=M_{h+1}^{*}$. Choose a positive number $M^{\prime \prime}$ such that $M^{\prime}<M^{\prime \prime}<M_{h+1}^{*}$. Consider in $G$ the locus $\left|f(z)-w_{0}\right|=M^{\prime \prime}$. The analysis set forth by Seidel and Walsh $[3, \S 14]$ applied to the present situation shows that the locus $\left|f(z)-w_{0}\right|=M^{\prime \prime}$ consists of a set of Jordan curves $J_{i}$ (one or more in number not exceeding $j_{0}$ ) such that the total number of zeros (counted according to multiplicity) of $f(z)-w_{0}$ enclosed by them is $j_{0}$. Let $h^{*}(z)$ be an extremal function of class $H$, that is, a function of the set $H$ for which we have $\lambda=L(h)=M^{\prime}$. If we write

$$
Q(z)=h^{*}(z)-f(z)=\left\{h^{*}(z)-w_{0}\right\}-\left\{f(z)-w_{0}\right\},
$$

we have $|Q(z)|<M^{\prime \prime}$ everywhere in $G$. Then on each Jordan curve $J_{i}$ we have

$$
\left|f(z)-w_{0}\right|=M^{\prime \prime}, \quad|Q(z)|<M^{\prime \prime} .
$$

It follows by Rouche's Theorem [1, p. 6] that the function $\left\{f(z)-w_{0}\right\}$ $+Q(z)$ has precisely as many zeros interior to each $J_{i}$ as has $f(z)-w_{0}$. This means that $h^{*}(z)$ takes the value $w_{0}$ at least $h+1$ times in $G$, contrary to the hypothesis that $h^{*}(z)$ belongs to the set $H$. The contradiction thus reached proves that $L(h)$ can not be less than $M_{h+1}^{*}$.

When $h=1$, the class $H$ becomes the family of functions analytic and univalent in $G$; and Theorem 1 becomes a result in the problem of approximating to a multivalent function by univalent functions. Theorem 1 then has $L(1) \geqq M_{2}^{*}$. Moreover, when $k \geqq 2$, if we let $q$ denote the largest of the integers $j=2,3, \cdots, k$ for which $M_{j}=M_{2}^{*}$, then the proof used in Theorem 1 shows that every function $F(z)$ analytic in $G$ and such that

$$
|F(z)-f(z)| \leqq \mu<M_{2}^{*}, \quad \quad z \text { in } G,
$$

is at least $q$-valent in $G$.

5. Extremal functions. Questions come to mind in connection with Theorem 1. What is the precise value of $L(h)$ ? When is there a unique 
extremal function? When does the set of extremal functions include an identically constant function? How are the extremal functions $h^{*}(z)$ related to $R$ and $S$ and the $S_{j}$ ? The following three theorems provide a beginning toward the solutions of these problems. In Theorem 2 the term $k$-sheeted circle has the meaning given to it by Seidel and Walsh [3, p. 159].

THEOREM 2. If the Riemann configuration $R$ onto which $G$ is mapped by $w=f(z)$ is a $k$-sheeted circle having center (or centers) of affix $w_{0}$, then $L(h)=r^{*}$; and one extremal function is $h^{*}(z) \equiv w_{0}$.

Proof. The circular hull of $S$ is identical with the closure of $S$; and we have $M_{1}^{*}=M_{2}^{*}=\cdots=M_{k}^{*}=r^{*}$. Consequently, $L(h)=r^{*}$; and one $h^{*}(z)$ is the constant function $h^{*}(z) \equiv w_{0}$.

THEOREM 3. If there exists a function $f(z)$ for which $L(h)$ is less than $r^{*}$, then none of the extremal functions $h^{*}(z)$ is constant.

Proof. The existence of an identically constant function for which $\lambda=L(h)<r^{*}$ would require that $S$ have a circular hull of radius less than $r^{*}$.

THEOREM 4. If $f(z)$ is such that $L(h)=r^{*}$, then the set of extremal functions $h^{*}(z)$ includes just one identically constant function $h^{*}(z)$ $\equiv w_{H}$, where $w_{H}$ is the center of the circular hull of $S$. Conversely, if the set of extremal functions $h^{*}(z)$ includes an identically constant function, then $L(h)=r^{*}$ and the constant member of the set of extremal functions is unique and is $h^{*}(z) \equiv w_{H}$.

Proof. When $L(h)=r^{*}$, then as in Theorem 2 the function $h(z)$ $\equiv w_{H}$ is an extremal function $h^{*}(z)$. If there were another identically constant extremal $h^{*}(z) \equiv w_{Q} \neq w_{H}$, then the projection $S$ would have to be contained in both the regions $\left|w-w_{H}\right|<r^{*}$ and $\left|w-w_{Q}\right|<r^{*}$. This would lead to the conclusion that $S$ must have a circular hull of radius less than $r^{*}$, contrary to hypothesis.

Conversely, if the set of extremals $h^{*}(z)$ contains an identically constant function $h^{*}(z) \equiv w_{0}$, then $L(h)$ can not be less than $r^{*}$ by Theorem 3. Then by the first part of the present theorem it follows that the function $h^{*}(z) \equiv w_{0}$ is the unique constant member of the set of extremals.

\section{REFERENCES}

1. J. L. Walsh, Interpolation and approximation by rational functions in the complex domain, Amer. Math. Soc. Colloq. Publ., Vol. 20, 2d ed., Amer. Math. Soc., Providence, R. I., 1956. 
2. Paul Montel, Legons sur les fonctions univalentes ou multivalentes, GauthierVillars, Paris, 1933.

3. W. Seidel and J. L. Walsh, On the derivatives of functions analytic in the unit circle and their radii of univalence and of p-valence, Trans. Amer. Math. Soc. 52 (1942), 128-216.

4. Paul Montel, Familles normales de fonctions analytiques, Gauthier-Villars, Paris, 1927.

5. F. Hausdorf, Mengenlehre, 3d ed., Walter de Gruyter, Berlin, 1935.

UnION COLLBGE AND

The General Electric Company 